\title{
GroopM: An automated tool for the recovery of population genomes from related metagenomes
}

Metagenomic binning methods that leverage differential population abundances in microbial communities (differential coverage) are emerging as a complementary approach to conventional composition-based binning. Here we introduce GroopM, an automated binning tool that primarily uses differential coverage to obtain high fidelity population genomes from related metagenomes. We demonstrate the effectiveness of GroopM using synthetic and realworld metagenomes, and show that GroopM produces results comparable with more time consuming, labor-intensive methods. 
1 GroopM: An automated tool for the recovery of population genomes from related 2 metagenomes

3 Michael Imelfort ${ }^{1}$, Donovan Parks ${ }^{1}$, Ben J. Woodcroft ${ }^{1}$, Paul Dennis ${ }^{1}$, Philip Hugenholtz ${ }^{1}$ and 4 Gene W. Tyson ${ }^{1,2}$

$5{ }^{1}$ Australian Center for Ecogenomics (ACE), School of Chemistry and Molecular Biosciences, 6 The University of Queensland, St Lucia, QLD 4072, Australia.

$7 \quad{ }^{2}$ Advanced Water Management Center (AWMC), The University of Queensland, St Lucia, QLD 8 4072, Australia.

9 Corresponding Authors: Gene W. Tyson and Michael Imelfort.

10 Address:

11

12 Phone:

13 Email: Australian Center for Ecogenomics, Building 76, The University of Queensland, St Lucia, QLD 4072, Australia.

(+617) 33653829

g.tyson@uq.edu.au 


\section{Introduction}

15 Our ability to understand the function and evolution of microbial communities has been

16 hampered by an inability to culture most component species in any given ecosystem (Hugenholtz

17 et al. 1998). Metagenomics, the application of shotgun sequencing to environmental DNA, has

18 provided a means to bypass this cultivation bottleneck and obtain genomic data broadly 19 representative of a microbial community (Handelsman 2004). Historically it has not been 20 possible to assemble the genomes of component species from complex communities due to 21 insufficient sequence coverage, therefore tool development has largely focused on classification 22 algorithms that assign taxonomy to sequence fragments (including reads) based on sequence 23 composition (McHardy et al. 2006), homology (Huson et al. 2007), phylogenetic affiliation

24 (Krause et al. 2008; Wu \& Eisen 2008) or a combination of these approaches (Brady \& Salzberg 25 2009; MacDonald et al. 2012; Parks et al. 2011). The main limitation underlying these methods is 26 their reliance on reference databases with a skewed genomic representation of microbial diversity 27 (Hugenholtz 2002).

28 Recent technological advances have permitted cost-effective deep sequencing ( $>50$ Gbp) of 29 metagenomes providing the resolution necessary to obtain partial or near complete genomes from 30 rare populations $(<1 \%)$ in complex communities (Albertsen et al. 2013; Wrighton et al. 2012).

31 However, the task of binning anonymous assembled sequence fragments (contigs) from a 32 metagenome into groups representative of their source populations remains a significant 33 challenge. One approach for binning deep metagenomes has been to cluster contigs with similar 34 tetranucleotide frequencies using emergent self organizing maps (TF-ESOMs) (Wrighton et al. 35 2012) or interpolated markov models ( 36 methods is low binning accuracy of short contigs $(<2 \mathrm{Kbp})$ and contigs from related 37 microorganisms with similar tetranucleotide frequencies (Teeling et al. 2004a; Teeling et al. $38 \underline{2004 b})$.

39 New binning approaches have recently emerged that primarily use differential coverage patterns

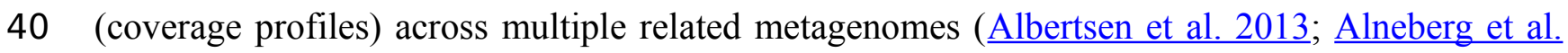
41 2013; Karlsson et al. 2013; Nielsen et al. 2014; Sharon et al. 2013). These approaches are based 42 on the assumption that contigs with similar coverage profiles are likely to have originated from 43 the same microbial population and show that combining coverage profiles with composition 
44 based approaches holds great promise for improving binning fidelity. Here we present GroopM, a

45 tool that automatically bins contigs into discrete population genomes based primarily on co-

46 varying coverage profiles across multiple related metagenomes, for example temporal or spatial

47 series of a given ecosystem.

48 Materials and Methods

49 Overview of the GroopM algorithm

50 The coverage profiles used by GroopM are generated by mapping reads from each related 51 metagenome onto an assembly of all or part of the same data. Contigs generated by any of the 52 many popular short read assemblers (e.g. Velvet (Zerbino \& Birney 2008), SPAdes (Bankevich et 53 al. 2012), IDBA-UD (Peng et al. 2012), Ray Meta (Boisvert et al. 2012)) can be used as input for 54 GroopM binning. A typical GroopM workflow progresses through five stages: parse, core, refine, 55 recruit and extract (Fig. 1). In the parse stage, GroopM loads and stores the data and creates the 56 various profiles used in the other stages (e.g. coverage profiles). The second stage, core, produces 57 a collection of preliminary bins which can be optionally refined and expanded in the refine and 58 recruit stages respectively. Finally, the extract stage includes a number of printing and data 59 extraction tools, which can be used to move GroopM specific data (e.g. bin assignments) into 60 standard file formats (e.g. FASTA, csv) for use by other programs in downstream analyses. A 61 detailed description of each of these stages is provided in the supplementary methods. GroopM 62 also provides a number of plotting options that allow users to visualize the layout of their 63 assembly in the various profile spaces (e.g. coverage profile, tetranucleotide frequency). GroopM 64 is licensed under the GPL version 3 and is freely available at 65 www.github.com/minillinim/GroopM.

\section{GroopM validation: Comparison with the TF-ESOM method using synthetic data}

67 Simulating metagenomic reads.

68 The relative abundances of community members in the synthetic data used in this comparison 69 was modeled on microbial community profiles (16S rRNA gene 97\% operational taxonomic unit $70[\mathrm{OTU}]$ tables) from three related soil samples from Stordalen mire (Woodcroft et al. 2013). The 71 OTU table generated from these samples contained 1,159 unique OTUs. Error-free 100bp paired72 end reads were generated from fully sequenced and permanent draft genomes (reference 73 sequences) which were downloaded from the Integrated Microbial Genomes (IMG) 74 (http://img.jgi.doe.gov) database, version 4.0 (See Supplementary Note 1). 
75 The metagenomic_mincer package (https://github.com/wwood/metagenomic_mincer) was used 76 to filter this collection of references and to create the synthetic reads. Oversampling of lineages 77 that have been the subject of concentrated sequencing efforts was avoided using the 78 remove_strains script in this package. The script detects when strains are from the same species 79 by searching for perfect matches in species name fields in the IMG metadata. Note that this 80 filtering strips most strain heterogeneity from the synthetic data resulting in metagenomes with 81 substantially simplified microbial community diversity and resulting assemblies that include

82 fewer and longer contigs than would be expected for assemblies of a typical soil metagenome. 83 The mince script was used to match reference sequences to OTUs and their corresponding 84 abundance levels. For each OTU present in the table, a bacterial or archaeal genome was chosen 85 at random from the filtered IMG reference set and synthetic reads were generated in accordance 86 with the corresponding sample relative abundance. Reads were generated using sammy 87 (https://github.com/minillinim/sammy) through the sammy_runner script in 88 metagenomic_mincer. In total, 128,229,878, 121,497,178 and 114,931,846 reads were generated 89 for each sample respectively (Supplementary Data). Data is available at 90 https://github.com/minillinim/GroopM_test_data/.

91 Assembly of synthetic reads using Velvet

92 The synthetic reads were co-assembled using Velvet version 1.2.07 (Zerbino \& Birney 2008) with 93 a kmer size of $85 \mathrm{bp}$ and an estimated insert size of $500 \mathrm{bp}$. The expected coverage and coverage 94 cutoff parameters were determined automatically by Velvet. After assembly, all contigs shorter 95 than $500 \mathrm{bp}$ were removed leaving 5,668 contigs totaling $133 \mathrm{Mb}$, with a longest contig of $961,667,234 \mathrm{bp}$ and an N50 of 122,645 bp. These contigs are referred to below as filtered contigs.

\section{Defining verified bin assignments}

98 The verified bin assignments for each filtered contig were determined by alignment to the 1,159

99 IMG reference genomes used to generate the data using BLAST version 2.2.25+ (Camacho et al. 100 2009). Almost all (>99\%) of the filtered contigs aligned to their corresponding reference 101 sequences with no mismatches. A total of 305 reference genomes were identified as the best 102 match for at least one filtered contig indicating that perfect binning of the filtered contigs should 103 produce 305 genome bins. The largest number of contigs mapped to a single reference was 696 
104 for Emticicia oligotrophica GPTSA100. Only 69 reference genomes were the best match for at

105 least 10 contigs and only 71 were assembled into contigs totaling at least $25 \mathrm{Kbp}$.

106 Reduction of bin counts to account for chimeric contigs

107 During the calculation of the verified bin assignments described above it was observed that a

108 number of contigs mapped equally well to multiple closely related reference genomes. The 109 reference genomes in question form two groups: The first group contains five genomes of the 110 genus Thermotoga; T. maritima MSB8, T. petrophila RKU-1, T. neapolitana DSM 4359, 111 Thermotoga sp. RQ2 and T. naphthophila RKU-10. The second consists of two strains of the 112 species Oenococcus oeni, PSU-1 and DSM 20252. These genomes were not labeled as strains 113 during data creation due to an error in the metadata. An analysis of read mapping data revealed 114 that $93.1 \%$ of reads generated from the O. oeni PSU-1 strain mapped to the DSM 20252 strain. 115 Similar results were found for reads generated for some of the Thermotoga genomes. These high 116 percentages of shared reads indicate that contigs mapping to these reference sequences could 117 have been chimerically constructed from reads generated from a mixture of reference sequences. 118 Therefore, it was decided that the two Oenococcus and the five Thermotoga variants should be 119 treated as two single populations leaving 300 verified bins after collapsing strains.

\section{Binning the filtered contigs using the TF-ESOM approach}

121 TF-ESOM binning was performed using Databionics ESOM tools (http://databionic122 esom.sourceforge.net) as described previously (Wrighton et al. 2012). Briefly, the frequencies of 123 all possible tetranucleotide sequences were calculated using a custom Perl script with a $1 \mathrm{bp}$ 124 sliding window that summed pairs of reverse complementary tetranucleotides. Contigs longer 125 than $5 \mathrm{Kbp}$ were split into $5 \mathrm{Kbp}$ fragments and contigs shorter than $2 \mathrm{Kbp}$ were excluded. 126 Tetranucleotide frequencies were normalized by contig length and application of the 'Robust ZT' 127 transformation built into ESOM tools. TF-ESOMs were toroidal and used Euclidean grid 128 distances and dimensions scaled from the default map size $(50 \times 82)$ as a function of the number 129 of data points, to a ratio of approximately 5.5 map nodes per data point. The Batch algorithm $(\mathrm{k}$ $130=0.15 \%, 20$ epochs) was used for training and the standard best match search method was used 131 with local best match search radius of 8 . Remaining training parameters were as follows: 132 Euclidean data space function; starting value for training radius of 50 with linear cooling to 1;

133 Gaussian weight initialization method; starting value for learning rate of 0.5 with linear cooling 134 to 0.1; Gaussian kernel function. Data points were assigned to classification groups (genome 
135 bins) by manually identifying the boundaries that were apparent using a distance-based

136 background topology (U-Matrix) representation of the TF-ESOM. Data points between bins or on

137 borders were not assigned to bins (Supplementary Fig. 1b).

\section{Binning the filtered contigs using GroopM}

139 Synthetic reads were mapped onto the filtered contigs using BWA version 0.6.2-r126 ( $\underline{\operatorname{Li} \&}$ 140 Durbin 2009) with default settings to produce three BAM files. The resulting BAM files were

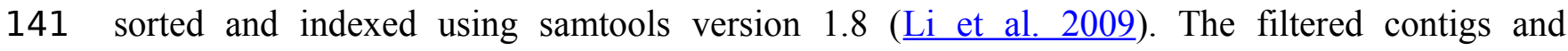
142 corresponding BAM files were parsed into GroopM version 0.2.0 and coring was carried out for 143 all contigs that were at least $1 \mathrm{Kbp}$ long, resulting in the formation of 63 core bins. Binned 144 contigs were saved to disk using GroopM extract and evaluated for contamination and 145 completeness based on the presence of 111 single copy marker genes (Dupont et al. 2011) using 146 CheckM (http://ecogenomics.github.io/CheckM/). The results of this analysis were used during

147 the running of GroopM refine to guide splitting and merging operations. First, bins with the 148 lowest completion scores were examined using GroopM's visualization tools to see if any 149 mergers were possible, resulting in 12 merge operations and one subsequent split operation. Next, 150 bins with the highest contamination scores were examined using GroopM's visualization tools to 151 determine if they were chimeras, resulting in three split operations (one which divided a bin into 152 three parts), one merge operation and two bin deletions. These operations reduced the number of 153 bins to 53 . The resulting genome bins were expanded to include contigs $<1 \mathrm{Kbp}$ using GroopM 154 recruit. Binned contigs were saved to disk using GroopM extract and evaluated again for 155 contamination and completeness.

\section{Evaluating binning accuracy}

157 Bins obtained using both methods were compared with the set of 300 verified bins described 158 above using the following method: First, each bin was assigned to the verified bin that contained 159 the majority of its contigs. Whenever two or more bins could be assigned to the same verified

160 bin, the competing bins were ranked in descending order of the cumulative length of contigs 161 belonging to the verified bin and only the top ranked bin was assigned to the verified bin. All 162 other bins were assigned to the verified bin that the next largest number of their contigs belonged 163 to. This procedure was applied iteratively until such time that each TF-ESOM and GroopM bin 164 was coupled with exactly one verified bin and that no verified bin was coupled with more than 165 one TF-ESOM or GroopM bin. Contigs were then classified as assigned correctly, incorrectly or 166 not assigned to any bin. Binned contigs that belonged to the same verified bin that their 
167 respective bin was coupled with were classified as binned correctly. All other binned contigs were

168 classified as binned incorrectly. All bins were evaluated for contamination and completeness

169 based on the presence of 111 single copy marker genes (Dupont et al. 2011) using CheckM

170 (Supplementary Tables 1 and 2).

\section{Recovery of population genomes from a human gut microbiome}

\section{Data preparation}

173 Unassembled data consisting of 18 paired Illumina short read data files sequenced from 11 fecal 174 samples was downloaded from the NCBI Sequence Read Archive 175 (http://www.ncbi.nlm.nih.gov/sra) on the 29th of May 2013. The corresponding binned contigs 176 (haron et al. 2013) (Sharon assembly) were downloaded from (http://ggkbase.berkeley.edu/) on 177 the 28th of May 2013 comprising 2,998 contigs separated into 33 groups, two of which appear to 178 be associated with contigs which could not be classified (CARUNCL and ACDRDN).

179 The published assembly process contained a number of tasks that would normally be categorized 180 as binning, comprising numerous iterations including a number of steps that focused on 181 identifying and obtaining genomes that differed at the strain level. In order to provide an 182 unbiased test of GroopM using strictly unbinned contigs, the raw data was re-assembled prior to 183 binning. Prior to assembly all reads were hard trimmed to $85 \mathrm{bp}$. Reads containing any sequence 184 beginning with three or more bases with quality score 2 were trimmed from the start of this 185 sequence until the end of the read. Finally, all reads shorter than 50bp were removed along with 186 their corresponding paired read.

187 Assembly and binning of the Sharon dataset using SPAdes and GroopM

188 The trimmed reads were assembled with SPAdes 2.4.0 (Bankevich et al. 2012) using default 189 parameters. As this version of SPAdes cannot handle 18 separate data files, all of the trimmed 190 reads files were concatenated to produce a single paired read data set. The resulting assembly was 191 filtered to remove all contigs shorter than $500 \mathrm{bp}$ leaving 7,016 contigs totaling $38.7 \mathrm{Mb}$, with a 192 longest contig of $406 \mathrm{Kbp}$ and an N50 of 36 Kbp. Trimmed reads were mapped onto the SPAdes 193 contigs with bwa-mem and the resulting output was parsed into GroopM using the same 194 approaches and software versions described above for the synthetic data assembly. GroopM 195 produced 24 core bins using all contigs that were at least $1 \mathrm{Kbp}$ long. Assessment of bin quality, 196 bin refinement and contig recruitment were performed using the method described above 
197 resulting in a single split operation, raising the total number of bins to 25 . Assembled contigs are 198 available at https://github.com/minillinim/GroopM_test_data.

199 Visualizing the Sharon assembly using GroopM

200 The trimmed reads were mapped onto the Sharon assembly using bwa-mem 0.7.5a ( $\underline{\operatorname{Li}} 2013)$ and

201 the resulting 18 BAM files were sorted and indexed using samtools 1.8 ( $\mathrm{Li}$ et al. 2009). The 202 Sharon assembly and corresponding BAM files were parsed into GroopM version 0.2.0 and the 203 resulting GroopM database was modified to incorporate the Sharon assembly bin assignments 204 allowing visualization using GroopM.

\section{Matching GroopM bins with the Sharon assembly}

206 Each SPAdes contig was linked to at most one of the contigs from the Sharon assembly using 207 nucmer version 3.07 (Delcher et al. 2002). Reciprocal best hit linkages were computed with a 208 custom python script (http://github.com/minillinim/taintedSwallow). A minority of SPAdes 209 contigs could not be linked to a Sharon contig and vice versa, however this is to be expected as 210 the two approaches used substantially different assembly approaches.

\section{Assessing the effects of using different assembly algorithms on GroopM output}

212 The trimmed Sharon data was assembled using three assemblers: SPAdes version 2.4.0 213 (Bankevich et al. 2012) (default parameters), CLC Bio version 7.0.3.64 (http://www.clcbio.com, 214 default parameters) and Velvet version 1.2.07 (Zerbino \& Birney 2008) (kmer 45bp). For each 215 assembly, all contigs $>=500 \mathrm{bp}$ were binned with GroopM using the process described above for 216 the synthetic data. Prodigal version 2.60 (Hyatt et al. 2010) (default parameters + meta switch) 217 was used to identify open reading frames (ORFs) in the Sharon bins and the three sets of GroopM 218 bins. The Sharon ORFs were aligned to each set of GroopM ORFs using Nucmer version 3.07 219 (Delcher et al. 2002) (default parameters) and the longest reciprocal match for each Sharon ORF 220 was determined using a custom python script (identity $>=99 \%$, length $>=60 \%$ ). The majority of

221 Sharon ORFs from each bin mapped to a single corresponding GroopM bin (dominant bin) with 222 the remainder mapping to several other bins. The similarity of GroopM outputs using different 223 assemblers was assessed using all Sharon bins with at least 1000 ORFs (11 bins).

\section{Results and Discussion}


225 We validated GroopM using synthetic metagenomes constructed from 1,159 reference genomes

226 with coverage patterns modeled on three related soil habitats. Co-assembly of these synthetic

227 metagenomes produced 5,668 contigs $(>0.5 \mathrm{Kbp})$, however, most genomes were present at low

228 coverage and did not assemble. Contigs were derived from 305 reference genomes (300 verified

229 bins), 71 of which each had a combined contig length of at least $25 \mathrm{Kbp}$ (Supplementary Data).

230 We compared bin assignments made using GroopM and TF-ESOM. Binning accuracy was 231 assessed based on agreement with verified bin assignments taking into account both total 232 numbers of contigs (TNC) and total assembled bases (TAB) that were assigned correctly, 233 incorrectly or not assigned to any bin. GroopM correctly binned $79.6 \%$ of the synthetic contigs 234 (97.5\% TAB), and performed substantially better than TF-ESOM (45.9\% TNC and 84.2\% TAB).

235 A much larger proportion of the metagenome was correctly binned with GroopM ( $\sim 73 \%$ more 236 contigs than TF-ESOM), and only $4.7 \%$ of the contigs were incorrectly binned $(0.4 \% \mathrm{TAB})$ as 237 opposed to $5.7 \%(8.7 \%$ TAB) for TF-ESOM. Furthermore, the GroopM errors were primarily 238 restricted to short contigs $(0.5$ to $2 \mathrm{Kbp})$ with only 62 contigs longer than $2 \mathrm{Kbp}$ incorrectly 239 assigned, including nine contigs between 10 to $30 \mathrm{Kbp}$. In contrast, TF-ESOM incorrectly 240 assigned 193 contigs longer than $5 \mathrm{Kbp}$, including 31 contigs longer than $100 \mathrm{Kbp}$ 241 (Supplementary Table 3).

242 We analyzed binning errors using visualizations of contig distributions and bin assignments in 243 coverage profile and tetranucleotide frequency spaces (Fig. 2). Differential coverage and 244 tetranucleotide frequency binning approaches may perform poorly in the presence of different 245 populations with highly similar coverage profiles and tetranucleotide frequencies, respectively. 246 As predicted, the majority of the GroopM errors (62.4\%) include populations with highly similar 247 coverage profiles (Figs. 2b, 2d and 2f), while most TF-ESOM errors $(81.3 \%)$ cluster around 248 populations with closely matching tetranucleotide frequencies (Figs. 2a, 2c and 2e). The majority 249 of GroopM errors were localized to seven genome bins (Supplementary Note 2), while the TF250 ESOM errors appeared more systemic, affecting 61 genome bins. The TF-ESOM errors were 251 mostly due to difficulties in identifying boundaries in ESOMs that separate contigs with highly 252 similar tetranucleotide frequency profiles (Supplementary Note 3). Our results show that 253 population genome bins can be more accurately resolved using coverage profiles augmented by 254 tetranucleotide frequencies than by using tetranucleotide frequencies alone (Fig. 3, 255 Supplementary Note 4). 
256 We further validated GroopM using 18 metagenomic datasets sourced from an infant human gut 257 microbiome previously analyzed by Sharon et al. (Sharon et al. 2013) using a custom iterative 258 assembly and binning process that included a coverage-based ESOM binning step. This produced 2592,998 contigs ( $>407$ bp) partitioned into 33 bins comprising microbial, viral and plasmid 260 genomes. To provide an unbiased test of GroopM, we reassembled the raw sequence data from 261 the 18 datasets using SPAdes (Bankevich et al. 2012) with default parameters producing 7,154 262 contigs ( $>500 \mathrm{bp})$. GroopM clustered 6,959 of these contigs $(99.5 \% \mathrm{TAB})$ into 25 population 263 genome bins using default parameters and minimal refinement steps. The completeness and 264 contamination of the Sharon and GroopM bins were estimated based on the presence and copy 265 number of 111 conserved single-copy bacterial marker genes (Dupont et al. 2011) using CheckM 266 (Supplementary Tables 4 and 5).

267 Overall, the two approaches produced highly similar population genome bins (Fig. 4). The six 268 most complete GroopM bins corresponded to the seven most complete Sharon bins, with all bins 269 having estimated completeness of greater than 70\% (Supplementary Tables 4 and 5). The 270 additional Sharon bin is one of two dominant Staphylococcus epidermidis strains that were co271 assembled and combined by GroopM into a single bin (GM_76). While GroopM did not 272 reconcile individual $S$. epidermidis strains, it grouped all of the contigs associated with the strains 273 together into a small number of bins suitable for manual refinement (Supplementary Note 5). 274 GroopM produced a number of genome bins which were more complete than those originally 275 reported, including two Propionibacterium population genomes: GM_11 (2.76 Mb, 89.2\% 276 complete) and GM_19 (1.1 Mb, 13.5\% complete) that correspond to Sharon bins CARPRO (2.52 $277 \mathrm{Mb}, 88.3 \%$ complete) and CARPAC (0.36 Mb, 2.7\% complete) respectively (Supplementary

278 Fig. 2 and Supplementary Note 6). The most striking example of genome recovery differences 279 was the complete absence of a partial GroopM bin (GM_34, 73 contigs, 75.6 Kbp, 39\% GC) in 280 the original study that was not detected in their contigs (Supplementary Figs. 3 and 4). Analysis 281 of alignments of contigs from this bin to IMG reference genomes indicates that GM_34 is most 282 likely a member of the Proteobacteria (Supplementary Data). The GM_34 population appears 283 to spike in abundance at day 21 , but is present at relatively low levels in all the other samples. It 284 is possible that the lack of good representation in multiple samples may have resulted in its poor 285 assembly. We speculate that differences in genome bin recovery are due to the iterative assembly 286 method used to create the Sharon contigs which may have filtered reads from some genome bins 287 resulting in partial or complete absences in the final assembly. Therefore caution should be 
288 exercised when using methods that involve strict filtering of the data, especially if the goal of the 289 analysis is to infer that the resulting population bins or microbial communities are missing key 290 metabolic components. Despite these and a small number of other minor binning differences 291 (Supplementary Tables 4 and 5), GroopM was able to produce highly similar population bins to 292 Sharon et al. (Sharon et al. 2013) in less than 2 hours with a small memory footprint 293 (Supplementary Table 6). Moreover, the GroopM binning was based on a single assembly of the 294 metagenomic data using default parameters, as opposed to the numerous assemblies and filtering 295 steps described in the original study.

296 We established that GroopM is not substantially affected by choice of assembler by repeating the 297 binning on two additional generic assemblies using Velvet and CLC and analyzing open reading 298 frame (ORF) distributions in the Sharon and GroopM bins. The CLC Bio and SPAdes assemblies 299 were very similar (SPAdes: 7,016 contigs, total $\sim 38.7 \mathrm{Mbp}$, N50 35,986bp, CLC: 7,576 contigs, 300 total $\sim 37.7 \mathrm{Mbp}$, N50 29,979bp) (Supplementary Fig. 5), however the Velvet assembly was sub301 optimal (7,199 contigs, total $\sim 29.7 \mathrm{Mbp}, \mathrm{N} 508,272 \mathrm{bp}$ ) and only included $\sim 75 \%$ of the bases 302 included in the Sharon, CLC Bio and SPAdes assemblies. Interestingly, the missing data from the 303 Velvet assembly originated from a small number of microorganisms whose contigs were almost 304 completely missing from the assembly. Of the contigs that Velvet did produce, the binning was 305 consistent with the CLC Bio and SPAdes-derived bins. We hypothesize that this could be the 306 result of Velvet's reliance on coverage cutoffs and highlights the motivation behind the multiple 307 assembly and filtering steps used to create the assembly published by Sharon et al. The 308 consistency of binning outcomes indicates that GroopM's output is not markedly affected by the 309 choice of assembly algorithm used, provided that the chosen assembler produces an "adequate" 310 assembly. The low proportion of matching ORFs for the CARSEP and CARSEP3 bins is the 311 result of GroopM's inability to reliably resolve bins at the strain level (Supplementary Note 2).

312 GroopM consistently groups most of the contigs from CARSEP and CARSEP3 together into a

313 single bin whose ORFs are almost evenly divided between the two Sharon bins. The similarity of 314 same strain groupings for all three assemblies provides further evidence that GroopM's output is 315 largely independent of the choice of assembler used.

\section{Conclusions}

317 In summary, GroopM automates production of high fidelity population genome bins from related 318 metagenomes by primarily leveraging differential coverage profiles. Decreasing sequencing costs 
319 and the push for increased replication (Prosser 2010) will make this a feasible approach for many

320 metagenomic projects. GroopM can bin contigs that have been generated using a range of 321 assembly methods including co-assemblies and single sample assemblies, provided that 322 metagenomic data is available for at least three related samples (Supplementary Note 7).

323 GroopM also provides a dedicated tool for visual interactive metagenomic bin editing. The 324 software allows users to view and merge bins, as well as split bins based on composition, 325 coverage or contig length profiles. Resolving population bins of closely related co-habiting 326 genotypes remains a challenge for all binning approaches. The current implementation of 327 GroopM places contigs from closely related genotypes into chimeric bins, as seen in the Sharon 328 et al. dataset (Supplementary Note 5), which require manual curation to separate.

329 During the review of this manuscript, we became aware of two studies that use similar coverage330 based approaches to bin population genomes from related metagenomic datasets. Nielsen et al. 331 (Nielsen et al. 2014) apply the method at the gene level, retrieving sets of genes that share co332 abundance profiles and Alneberg et al. (Alneberg et al. 2013) report a tool called CONCOCT that 333 uses a combines differential coverage and tetranucleotide frequency profiles with linking reads to

334 bin contigs into population genomes. It is beyond the scope of the present study to compare 335 GroopM to these methods, but assessing their relative strengths and weaknesses is clearly an 336 important objective for future studies.

337 GroopM is currently being used to recover hundreds of high fidelity population genomes from 338 numerous habitats including anaerobic digesters, permafrost and host-associated systems (human, 339 coral, insect, plant). Such genomes serve two important purposes. Firstly, they rapidly fill out the 340 microbial tree of life, in particular by providing the first genomic representation for many 341 candidate phyla, which is important for correcting our currently skewed understanding of 342 microbial evolution. Secondly, they provide a basis for development of genome-based trophic 343 interaction networks to facilitate understanding of how microbial communities function in given 344 ecosystems. These developments will ensure that microbiologists can make best use of the 345 opportunities presented by the ongoing high throughput sequencing revolution.

\section{Acknowledgements and author contributions}

347 MI, GWT and PH conceptually defined the project. MI designed the algorithm and wrote 348 GroopM. DP contributed to the development of GroopM, greatly improving portions of the 
349 algorithm. BW produced the synthetic data used in the validation of the algorithm and supplied 350 many helpful comments regarding both algorithm development and validation. PD carried out the 351 ESOM-based binning of the synthetic data. GWT and MI wrote the initial draft of the manuscript. 352 All authors contributed to the final drafts of the manuscript. We thank Harald Gruber-Vodicka 353 and an anonymous reviewer for their useful input.

\section{Competing interests}

355 The authors declare no competing financial or other interests. 


\section{References}

357

358

359

360

361

362

363

364

365

366

367

368

369

370

371

372

373

374

375

376

377

378

379

380

381

382

383

384

385

386

387

388

389

390

391

392

393

394

395

396

397

398

399

400

401

402

403

Albertsen M, Hugenholtz P, Skarshewski A, Nielsen KL, Tyson GW, and Nielsen PH. 2013. Genome sequences of rare, uncultured bacteria obtained by differential coverage binning of multiple metagenomes. Nature biotechnology 31:533-538.

Alneberg J, Bjarnason BS, de Bruijn I, Schirmer M, Quick J, Ijaz UZ, Loman NJ, Andersson AF, and Quince C. 2013. CONCOCT: Clustering cONtigs on COverage and ComposiTion. arXiv preprint arXiv:13124038.

Bankevich A, Nurk S, Antipov D, Gurevich AA, Dvorkin M, Kulikov AS, Lesin VM, Nikolenko SI, Pham S, and Prjibelski AD. 2012. SPAdes: A new genome assembly algorithm and its applications to single-cell sequencing. Journal of Computational Biology 19:455-477.

Boisvert S, Raymond F, Godzaridis E, Laviolette F, and Corbeil J. 2012. Ray Meta: scalable de novo metagenome assembly and profiling. Genome Biol 13:R122.

Brady A, and Salzberg SL. 2009. Phymm and PhymmBL: metagenomic phylogenetic classification with interpolated Markov models. Nature methods 6:673-676.

Camacho C, Coulouris G, Avagyan V, Ma N, Papadopoulos J, Bealer K, and Madden T. 2009. BLAST+: architecture and applications. BMC bioinformatics 10:421.

Delcher AL, Phillippy A, Carlton J, and Salzberg SL. 2002. Fast algorithms for large-scale genome alignment and comparison. Nucleic acids research 30:2478-2483.

Dupont CL, Rusch DB, Yooseph S, Lombardo M-J, Richter RA, Valas R, Novotny M, YeeGreenbaum J, Selengut JD, and Haft DH. 2011. Genomic insights to SAR86, an abundant and uncultivated marine bacterial lineage. The ISME journal 6:1186-1199.

Handelsman J. 2004. Metagenomics: application of genomics to uncultured microorganisms. Microbiology and Molecular Biology Reviews 68:669-685.

Hugenholtz P. 2002. Exploring prokaryotic diversity in the genomic era. Genome Biol 3:10003.0008 .

Hugenholtz P, Goebel BM, and Pace NR. 1998. Impact of culture-independent studies on the emerging phylogenetic view of bacterial diversity. Journal of bacteriology 180:47654774.

Huson DH, Auch AF, Qi J, and Schuster SC. 2007. MEGAN analysis of metagenomic data. Genome research 17:377-386.

Hyatt D, Chen G-L, LoCascio PF, Land ML, Larimer FW, and Hauser LJ. 2010. Prodigal: prokaryotic gene recognition and translation initiation site identification. $B M C$ bioinformatics 11:119.

Karlsson FH, Tremaroli V, Nookaew I, Bergström G, Behre CJ, Fagerberg B, Nielsen J, and Bäckhed F. 2013. Gut metagenome in European women with normal, impaired and diabetic glucose control. Nature 498:99-103.

Krause L, Diaz NN, Goesmann A, Kelley S, Nattkemper TW, Rohwer F, Edwards RA, and Stoye J. 2008. Phylogenetic classification of short environmental DNA fragments. Nucleic acids research 36:2230-2239.

Li H. 2013. Aligning sequence reads, clone sequences and assembly contigs with BWA-MEM. arXiv preprint arXiv: 13033997.

Li H, and Durbin R. 2009. Fast and accurate short read alignment with Burrows-Wheeler transform. Bioinformatics 25:1754-1760.

Li H, Handsaker B, Wysoker A, Fennell T, Ruan J, Homer N, Marth G, Abecasis G, and Durbin R. 2009. The sequence alignment/map format and SAMtools. Bioinformatics 25:20782079.

MacDonald NJ, Parks DH, and Beiko RG. 2012. Rapid identification of high-confidence taxonomic assignments for metagenomic data. Nucleic acids research 40:e111-e111.

PeerJ reviewing PDF | (v2014:06:2209:2:0:NEW 5 Aug 2014) 
404

405

406

407

408

409

410

411

412

413

414

415

416

417

418

419

420

421

422

423

424

425

426

427

428

429

430

431

432

433

434

435

436

437

438

439

440

441

442

443

McHardy AC, Martin HG, Tsirigos A, Hugenholtz P, and Rigoutsos I. 2006. Accurate phylogenetic classification of variable-length DNA fragments. Nature methods 4:63-72. Nielsen HB, Almeida M, Juncker AS, Rasmussen S, Li J, Sunagawa S, Plichta DR, Gautier L, Pedersen AG, Le Chatelier E, Pelletier E, Bonde I, Nielsen T, Manichanh C, Arumugam M, Batto J-M, Quintanilha dos Santos MB, Blom N, Borruel N, Burgdorf KS, Boumezbeur F, Casellas F, Dore J, Dworzynski P, Guarner F, Hansen T, Hildebrand F, Kaas RS, Kennedy S, Kristiansen K, Kultima JR, Leonard P, Levenez F, Lund O, Moumen B, Le Paslier D, Pons N, Pedersen O, Prifti E, Qin J, Raes J, Sorensen S, Tap J, Tims S, Ussery DW, Yamada T, Meta HITC, Renault P, Sicheritz-Ponten T, Bork P, Wang J, Brunak S, and Ehrlich SD. 2014. Identification and assembly of genomes and genetic elements in complex metagenomic samples without using reference genomes. Nat Biotech advance online publication.

Parks DH, MacDonald NJ, and Beiko RG. 2011. Classifying short genomic fragments from novel lineages using composition and homology. BMC bioinformatics 12:328.

Peng Y, Leung HC, Yiu S-M, and Chin FY. 2012. IDBA-UD: a de novo assembler for single-cell and metagenomic sequencing data with highly uneven depth. Bioinformatics 28:14201428.

Prosser JI. 2010. Replicate or lie. Environmental microbiology 12:1806-1810.

Sharon I, Morowitz MJ, Thomas BC, Costello EK, Relman DA, and Banfield JF. 2013. Time series community genomics analysis reveals rapid shifts in bacterial species, strains, and phage during infant gut colonization. Genome research 23:111-120.

Strous M, Kraft B, Bisdorf R, and Tegetmeyer HE. 2012. The binning of metagenomic contigs for microbial physiology of mixed cultures. Frontiers in microbiology 3.

Teeling H, Meyerdierks A, Bauer M, Amann R, and Glöckner FO. 2004a. Application of tetranucleotide frequencies for the assignment of genomic fragments. Environmental microbiology 6:938-947.

Teeling H, Waldmann J, Lombardot T, Bauer M, and Glöckner FO. 2004b. TETRA: a webservice and a stand-alone program for the analysis and comparison of tetranucleotide usage patterns in DNA sequences. BMC bioinformatics 5:163.

Woodcroft BJ, Mondav R, Kim E-H, McCalley CM, Hodgkins SB, Crill PM, Chanton J, Hurst GB, VerBerkmoes NC, Saleska SR, Hugenholtz P, Rich VI, and Tyson GW. 2013. Discovery of a novel methanogen prevalent in thawing permafrost. Nature Communications.

Wrighton KC, Thomas BC, Sharon I, Miller CS, Castelle CJ, VerBerkmoes NC, Wilkins MJ, Hettich RL, Lipton MS, and Williams KH. 2012. Fermentation, hydrogen, and sulfur metabolism in multiple uncultivated bacterial phyla. Science 337:1661-1665.

Wu M, and Eisen JA. 2008. A simple, fast, and accurate method of phylogenomic inference. Genome Biol 9:R151.

Zerbino DR, and Birney E. 2008. Velvet: algorithms for de novo short read assembly using de Bruijn graphs. Genome research 18:821-829. 


\section{Figure 1}

\section{An overview of the GroopM workflow.}

GroopM has five stages, beginning with file parsing and ending with bin extraction. The refine step is optional and can be carried out at any stage after "core" has completed.

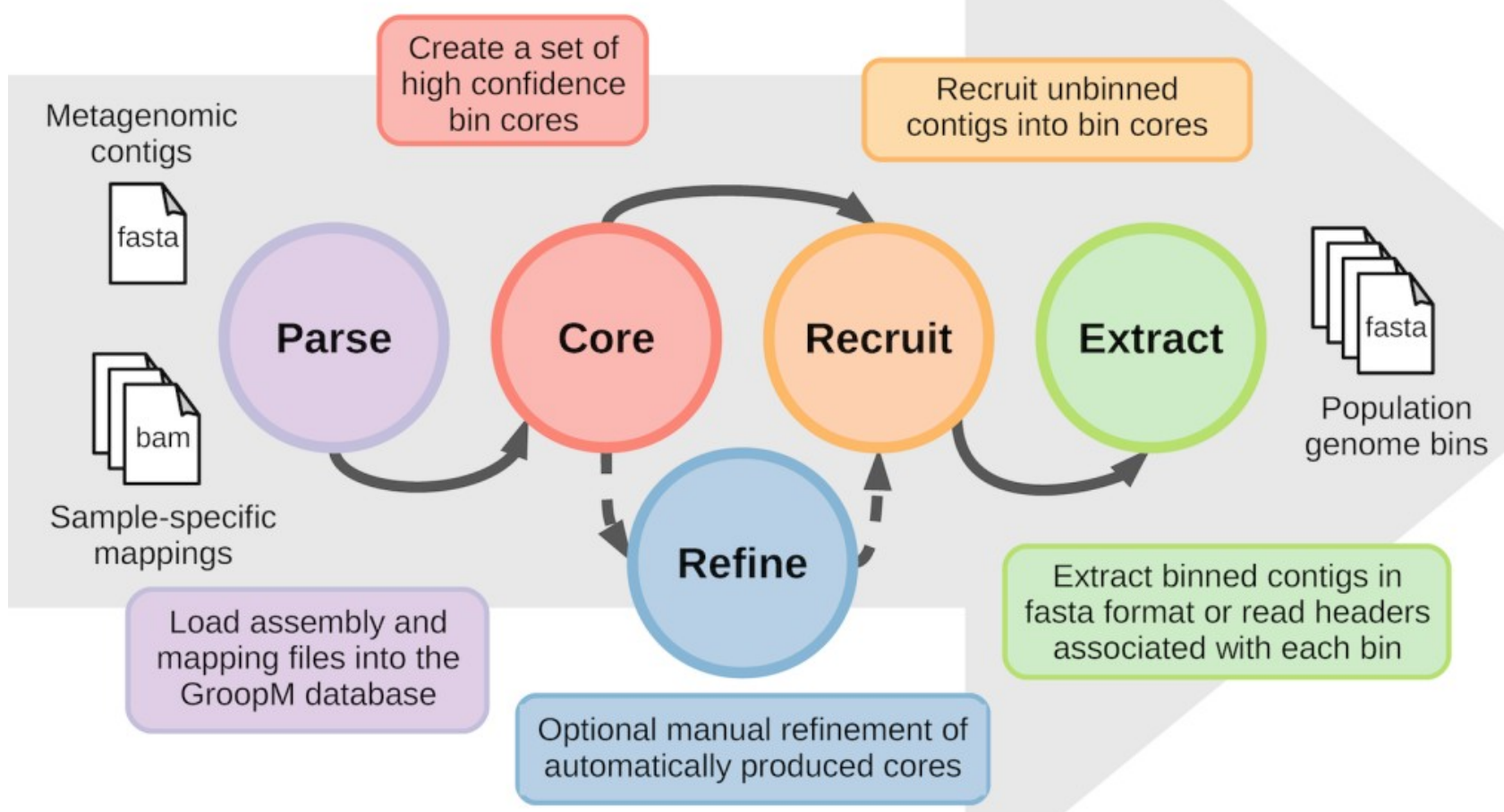




\section{Figure 2}

The distribution of tetranucleotide frequencies, coverage profiles and bin assignments for the synthetic metagenomic contigs.

The diameter of each circle is proportional to the length its respective contig. $(\mathbf{a}, \mathbf{c}, \mathbf{e})$ Contigs are positioned according to the first two principal components of their tetranucleotide frequencies. The first principal component is positioned horizontally, the second is positioned vertically. $(\mathbf{b}, \mathbf{d}, \mathbf{f})$ Contigs are positioned according to their $\mathrm{x}$ and $\mathrm{y}$ coordinates in GroopM transformed coverage profile space. $(\mathbf{a}, \mathbf{b})$ Each 'true' bin is assigned a random color and contigs are colored according to their true bin assignments. (c,d) Contigs are colored according to the accuracy of their bin assignments using TF-ESOM. $(e, f)$ Contigs are colored according to the accuracy of their bin assignments using GroopM. 
a

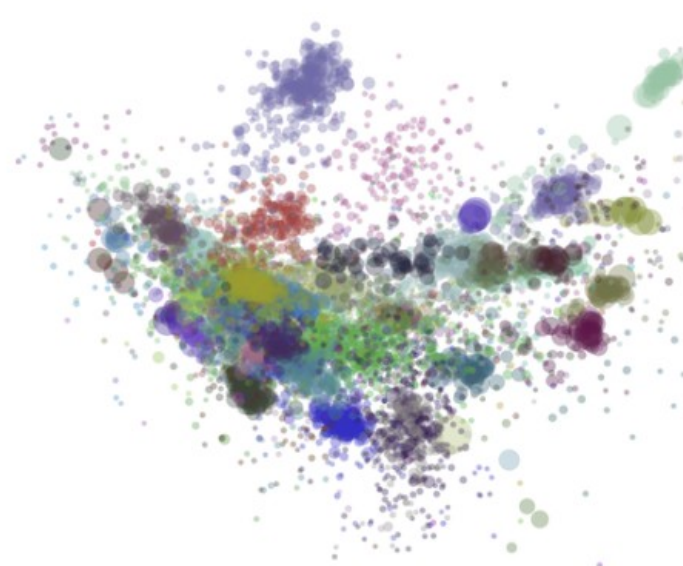

C

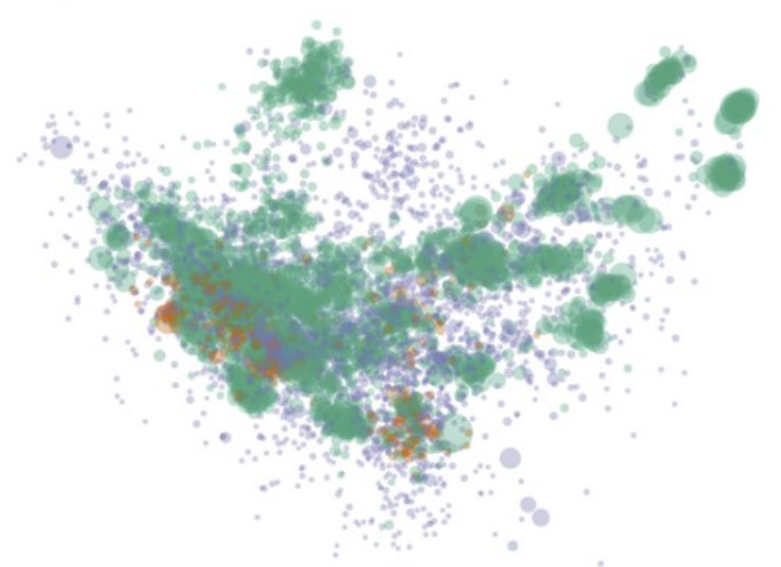

e

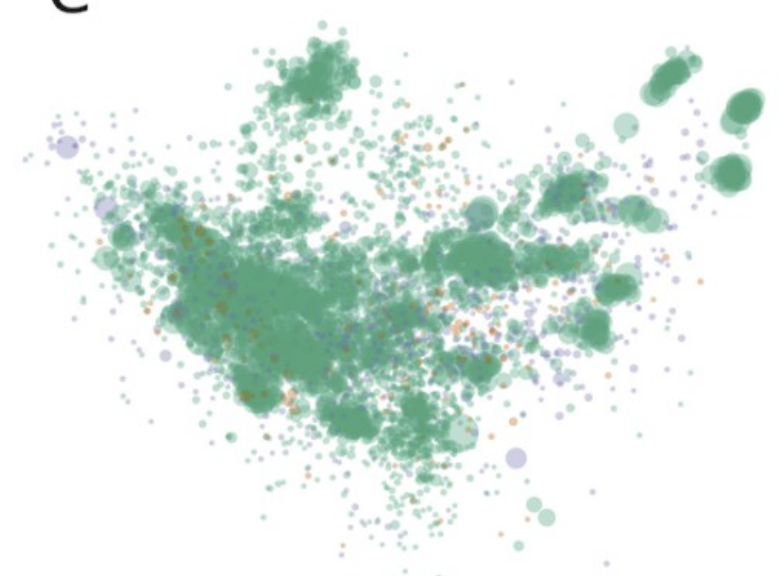

b

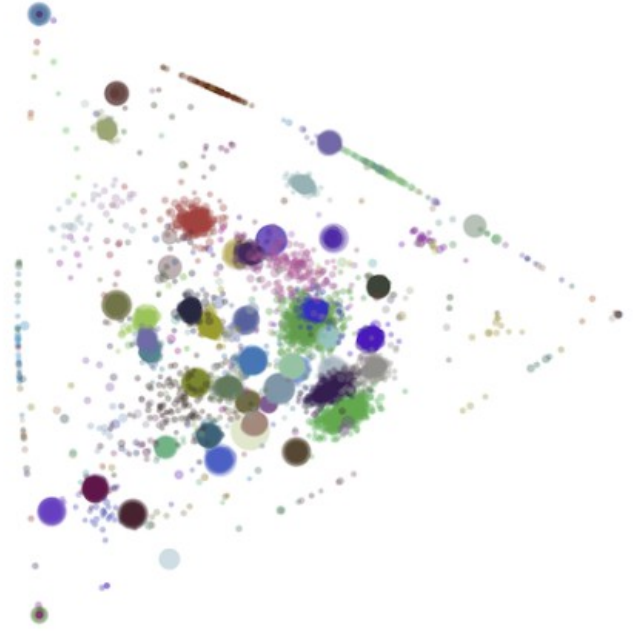

d

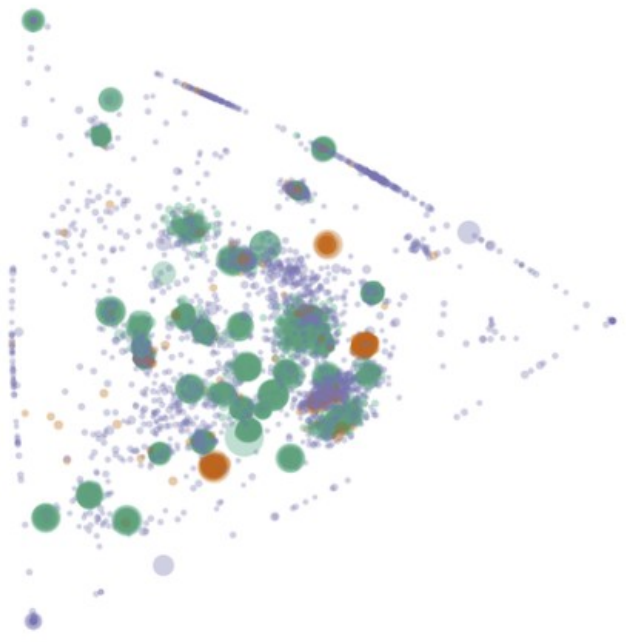

f

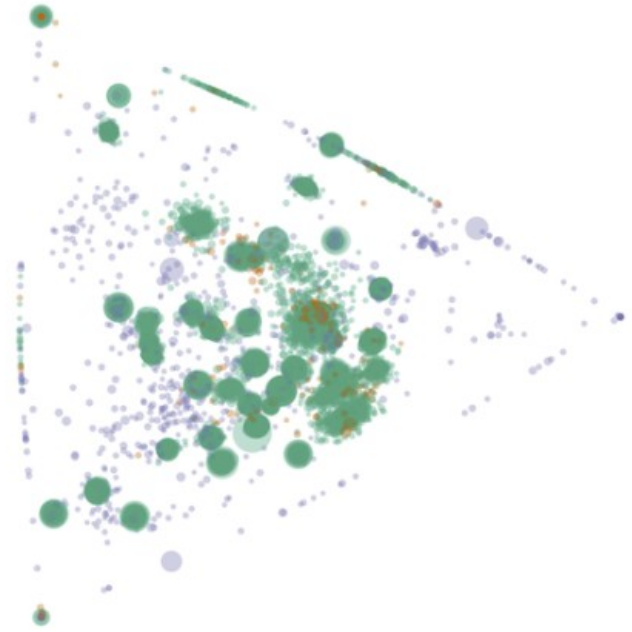




\section{Figure 3}

An overview of the relationships between contig length, population relative abundance and binning accuracy for the TF-ESOM and GroopM approaches.

(a) Contigs are ordered from longest to shortest and grouped together into clusters of 50. Each bar represents a single cluster and has a width that is proportional to the total number of assembled bases in that cluster. Bars are split vertically according to the percentage of their bases that are either correctly, incorrectly or not assigned. The large region of unassigned contigs in the TF-ESOM plot reflects the lower binning limit of $2 \mathrm{Kbp}$ for this method. (b) Verified bins were ordered in descending order of relative abundance, calculated based on the number of simulated reads created using each reference. Each bar represents a single verified bin and the height of each bar represents the bins relative abundance. Bars are split vertically according to the percentage of their bases that are either correctly, incorrectly or not assigned by the corresponding method. Both methods had decreased accuracy for very low abundance bins however GroopM was able to correctly bin nearly all the contigs for the most dominant species. 

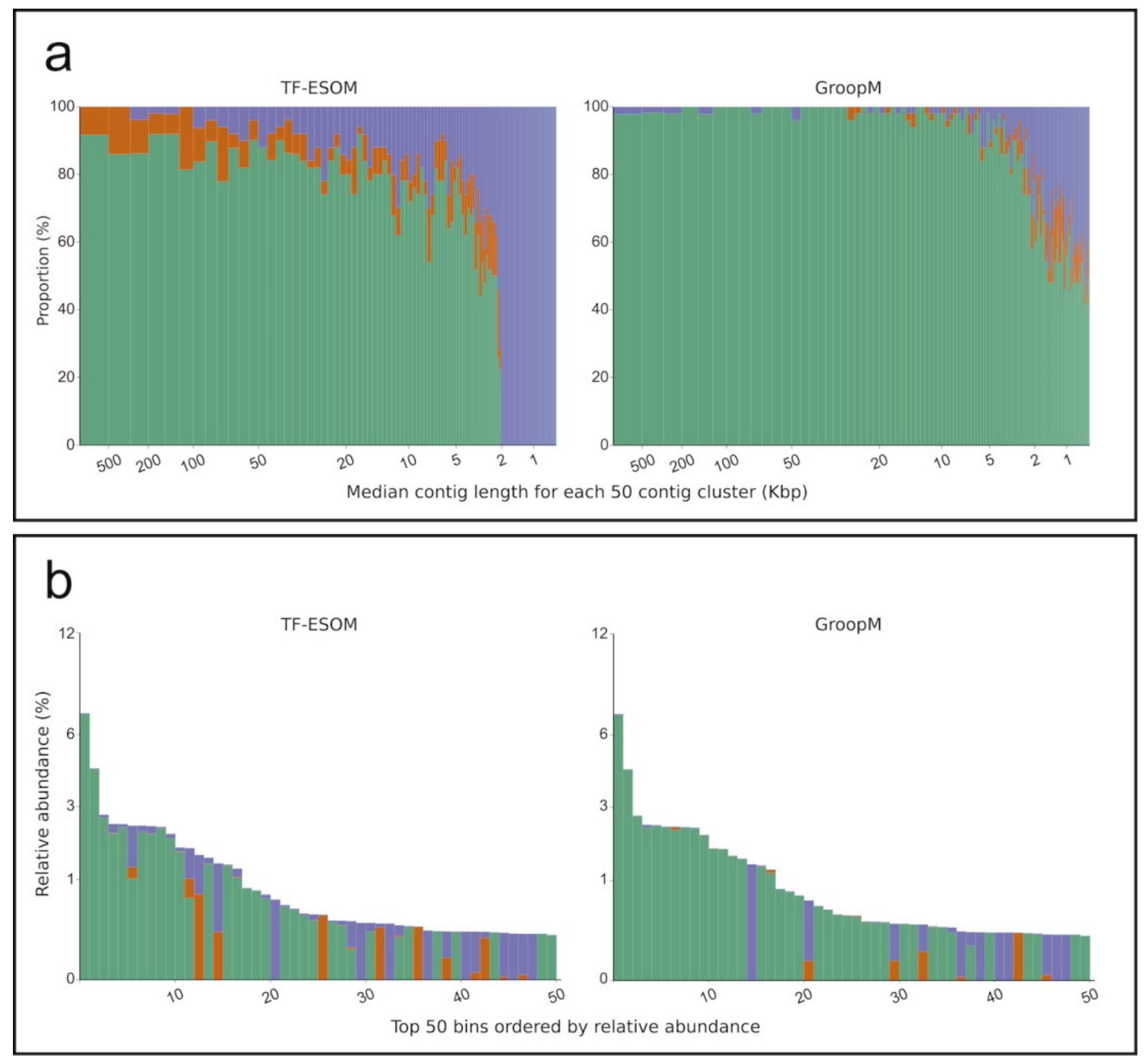

Correctly assigned

Incorrectly assigned

Unassigned 


\section{Figure 4}

A comparison of GroopM and Sharon bin assignments generated using visualization tools within GroopM.

(a) Contigs and resulting bins made using SPAdes and GroopM. (b) The Sharon assembly visualized in GroopM coverage space. All the contigs belonging to a single bin are assigned the same color. Each bin was assigned a random unique color with the exception of strain variants which were assigned very similar colors. GroopM-binned contigs are colored according to the bin assignment of their closest matching contig in the Sharon assembly.
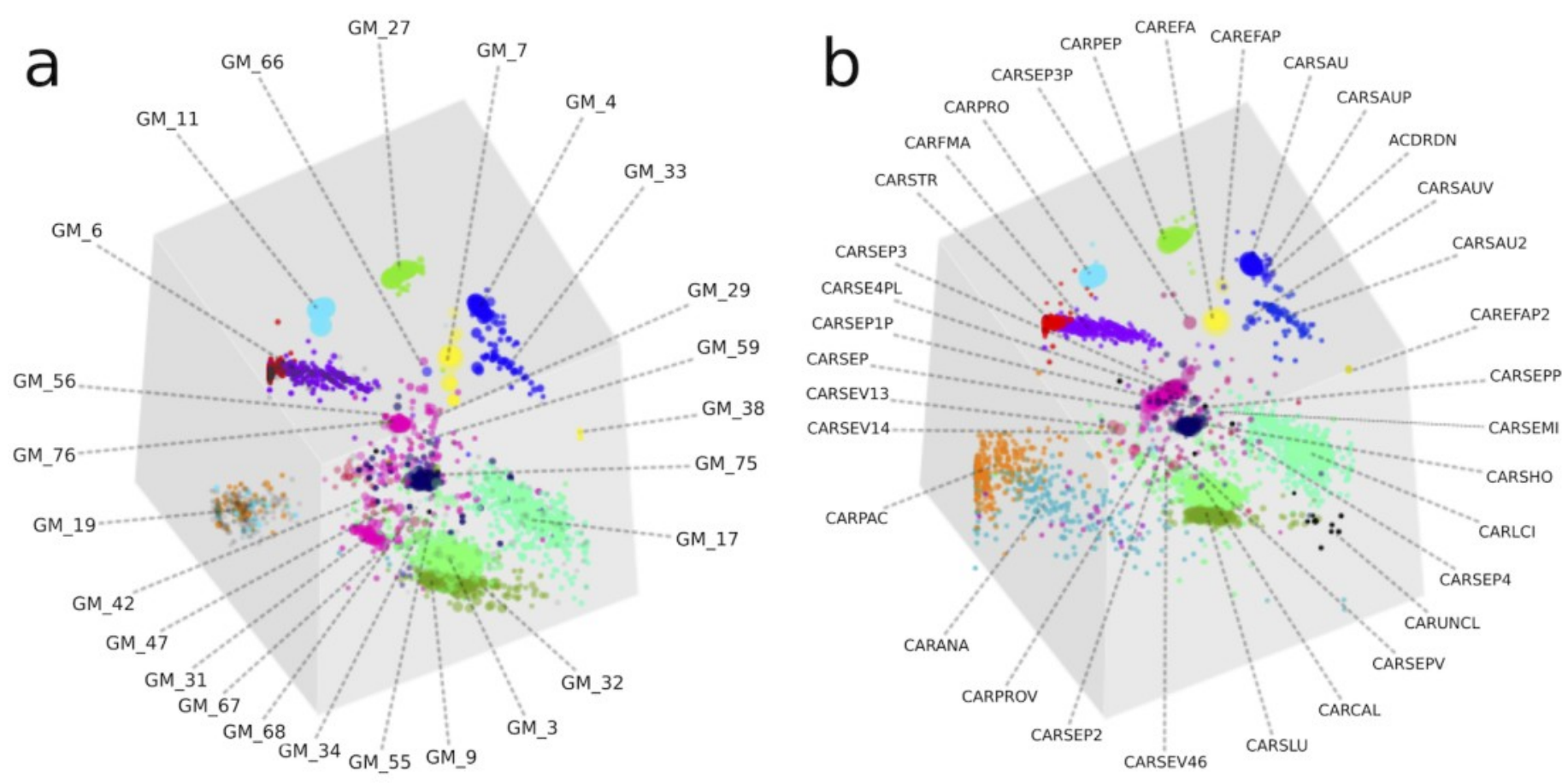